\title{
NEW LOWER BOUNDS FOR THE LEAST COMMON MULTIPLES OF ARITHMETIC PROGRESSIONS
}

\author{
RONGJUN WU, QIANRONG TAN, AND SHAOFANG HONG*
}

\begin{abstract}
For relatively prime positive integers $u_{0}$ and $r$ and for $0 \leq k \leq n$, define $u_{k}:=u_{0}+k r$. Let $L_{n}:=\operatorname{lcm}\left(u_{0}, u_{1}, \ldots, u_{n}\right)$ and let $a, l \geq 2$ be any integers. In this paper, we show that, for integers $\alpha \geq a$ and $r \geq \max (a, l-1)$ and $n \geq l \alpha r$, we have

$$
L_{n} \geq u_{0} r^{(l-1) \alpha+a-l}(r+1)^{n} .
$$
\end{abstract}

Particularly, letting $l=3$ yields an improvement to the best previous lower bound on $L_{n}$ obtained by Hong and Kominers.

\section{INTRODUCTION}

Hanson and Nair initiated the search for effective estimates for the least common multiple of the terms in a finite arithmetic progression; and, in [6] and in [13] they managed to produce good upper and lower bounds for $\operatorname{lcm}(1,2, \ldots, n)$. In particular, Nair 13 discovered a nice new proof for the following well-known nontrivial lower bound

$$
\operatorname{lcm}(1,2, \ldots, n) \geq 2^{n-1}
$$

for any integer $n \geq 1$. In [4, Farhi provided an identity involving the least common multiple of binomial coefficients and then use it to give a simple proof of the estimate (1.1). Inspired by Hanson's and Nair's works, Bateman, Kalb, and Stenger [1] and Farhi 2] respectively sought asymptotics and nontrivial lower bounds for the least common multiples of arithmetic progressions. Recently, Hong, Qian and Tan 10 extended the Bateman-Kalb-Stenger theorem from the linear polynomial to the product of linear polynomials. On the other hand, Farhi 2] obtained several nontrivial bounds and posed a conjecture which was later confirmed by Hong and Feng [7]. Hong and Feng [7] also got an improved lower bound for sufficiently long arithmetic progressions; this result was later sharpened further by Hong and Yang [11]. We notice that Hong and Yang [12] and Farhi and Kane [5] obtained some related results regarding the least common multiple of a finite number of consecutive integers. The theorem of Farhi and Kane 5 was extended by Hong and Qian 9] from the set of positive integers to the general arithmetic progression case. Recently, Qian, Tan and Hong [14] obtained some results about the least common multiple of consecutive terms in a quadratic progression.

In this paper, we study finite arithmetic progressions $\left\{u_{k}:=u_{0}+k r\right\}_{k=0}^{n}$ with $u_{0}, r \geq 1$ being integers satisfying $\left(u_{0}, r\right)=1$. Throughout, we define $L_{n}:=\operatorname{lcm}\left(u_{0}, u_{1}, \ldots, u_{n}\right)$ to be the least common multiple of the sequence $\left\{u_{k}\right\}_{k=0}^{n}$. We begin with the following

2000 Mathematics Subject Classification. Primary 11B25, 11N13, 11A05.

Key words and phrases. Arithmetic progression; least common multiple; lower bound.

*Hong is the corresponding author and was supported partially by the National Science Foundation of China Grant \# 10971145 and by the Ph.D. Programs Foundation of Ministry of Education of China Grant \#20100181110073. 
lower bound on $L_{n}$ :

Theorem 1.1. [11] Let $\alpha \geq 1$ be an integer. If $n>r^{\alpha}$, then we have $L_{n} \geq$ $u_{0} r^{\alpha}(r+1)^{n}$.

If $r=1$, then Theorem 1.1 is the conjecture of Farhi [2] proven by Hong and Feng [7]. If $\alpha=1$, then Theorem 1.1 becomes the improved lower bound of Hong and Feng [7. In [8], Hong and Kominers sharpened the lower bound in Theorem 1.1 whenever $\alpha, r \geq 2$. In particular, they proved the following theorem which replaces the exponential condition $n>r^{\alpha}$ of Theorem 1.1 with a linear condition $n \geq 2 \alpha r$.

Theorem 1.2. [8] Let $a \geq 2$ be any given integer. Then for any integers $\alpha, r \geq a$ and $n \geq 2 \alpha r$, we have $L_{n} \geq u_{0} r^{\alpha+a-2}(r+1)^{n}$.

Letting $a=2$, we see that Theorem 1.2 improves upon Theorem 1.1 for all but three choices of $\alpha, r \geq 2$. In the present paper, we provide a more general lower bound as follows.

Theorem 1.3. Let $a, l \geq 2$ be any given integers. Then for any integers $\alpha \geq a$ and $r \geq \max (a, l-1)$ and $n \geq l \alpha r$, we have $L_{n} \geq u_{0} r^{(l-1) \alpha+a-l}(r+1)^{n}$.

Picking $l=2$, then Theorem 1.3 becomes Theorem 1.2. Letting $l=3$ in Theorem 1.3 gives us the following new lower bound.

Theorem 1.4. Let $a \geq 2$ be any given integer. Then for any integers $\alpha, r \geq a$ and $n \geq 3 \alpha r$, we have $L_{n} \geq u_{0} r^{2 \alpha+a-3}(r+1)^{n}$.

Since $\alpha \geq a \geq 2$, we have $2 \alpha+a-3>\alpha+a-2$. Therefore the lower bound in Theorem 1.4 is better than that of Theorem 1.2 when $n$ is large enough.

This paper is organized as follows. In Section 2, we first introduce relevant notation and previous results. Finally, we prove Theorem 1.3.

\section{Proof of Theorem 1.3}

For any real numbers $x$ and $y$, we say that $y$ divides $x$ if there exists an integer $z$ such that $x=y z$. If $x$ divides $y$, then we write $y \mid x$. As usual, we let $\lfloor x\rfloor$ denote the largest integer no more than $x$.

Following Hong and Yang [11, we denote, for each integer $0 \leq k \leq n$,

$$
C_{n, k}:=\frac{u_{k} \cdots u_{n}}{(n-k) !}, \quad L_{n, k}:=\operatorname{lcm}\left(u_{k}, \ldots, u_{n}\right) .
$$

From the latter definition, we have that $L_{n}=L_{n, 0}$.

The following Lemma first appeared in [2] and was reproved in [3] and [7].

Lemma 2.1. 2] [3] 7] For any integer $n \geq 1, C_{n, 0} \mid L_{n}$.

From Lemma 2.1, we see immediately that

$$
L_{n, k}=A_{n, k} \frac{u_{k} \cdots u_{n}}{(n-k) !}=A_{n, k} \cdot C_{n, k}
$$


for some integer $A_{n, k} \geq 1$.

Following Hong and Feng [7] and Hong and Yang [11, we define, for any $n \geq 1$,

$$
k_{n}:=\max \left\{0,\left\lfloor\frac{n-u_{0}}{r+1}\right\rfloor+1\right\} .
$$

Hong and Feng [7] proved the following result.

Lemma 2.2. 7] For all $n \geq 1$ and $0 \leq k \leq n$,

$$
L_{n} \geq L_{n, k_{n}} \geq C_{n, k_{n}} \geq u_{0}(r+1)^{n} .
$$

Now we are in a position to prove a lemma whose proof closely follows the approach of Hong and Yang [1].

Lemma 2.3. Let $a, l \geq 2$ be any given integers. Then for any integers $\alpha \geq a$ and $r \geq \max (a, l-1)$ and $n \geq l \alpha r$, we have $n-k_{n}>((l-1) \alpha+a-l) r$.

Proof. If $n \leq u_{0}$, then by the definition (2.2), $k_{n} \leq 1$. Since $\alpha, r \geq a \geq 2$ and $n \geq l \alpha r$, we derive that $n-k_{n} \geq n-1 \geq l \alpha r-1>((l-1) \alpha+a-l) r$.

Now we suppose that $n>u_{0}$. In this case, we have

$$
k_{n}=\left\lfloor\frac{n-u_{0}}{r+1}\right\rfloor+1 \text {. }
$$

So we have

$$
k_{n} \leq \frac{n-u_{0}}{r+1}+1 \leq \frac{n-1}{r+1}+1=\frac{n+r}{r+1} .
$$

It then follows that

$$
n-k_{n} \geq n-\frac{n+r}{r+1}=\frac{(n-1) r}{r+1} \geq \frac{(l \alpha r-1) r}{r+1} .
$$

Note that $r \geq l-1$ tells us that $r-l+1 \geq 0$. Then from the assumption $\alpha, r \geq a$ it follows that

$$
\begin{aligned}
(l \alpha r-1)-(r+1)((l-1) \alpha+a-l) & =(r-l+1) \alpha-1-(r+1)(a-l) \\
& \geq a(r-l+1)-1-(r+1)(a-l) \\
& =l(r-a)+l-1>0 .
\end{aligned}
$$

Therefore by (2.4), we infer that

$$
\frac{l \alpha r-1}{r+1}>(l-1) \alpha+a-l .
$$

The desired result then follows immediately from (2.3) and (2.5).

Using the similar argument as that of Theorem 1.1, by Lemma 2.3 we can now prove Theorem 1.3 as the conclusion of this paper.

Proof of Theorem 1.3. By hypothesis, we have $\alpha, r \geq a \geq 2, l \geq 2$ and $n \geq l \alpha r$. It follows from Lemma 2.3 that $r^{(l-1) \alpha+a-l} \mid\left(n-k_{n}\right)$ !. Thus, we may express $\left(n-k_{n}\right)$ ! in the form $r^{(l-1) \alpha+a-l} \cdot B_{n}=\left(n-k_{n}\right)$ !, with $B_{n} \geq 1$ being an integer. Letting $k=k_{n}$ in (2.1), we find that

$$
r^{(l-1) \alpha+a-l} \cdot B_{n} \cdot L_{n, k_{n}}=A_{n, k_{n}} \cdot u_{k_{n}} \cdots u_{n} .
$$


It then follows that $r^{(l-1) \alpha+a-l} \mid A_{n, k_{n}}$, since the requirement $\left(r, u_{0}\right)=1$ implies that $\left(r, u_{k}\right)=1$ for all $0 \leq k \leq n$. Then, we get from (2.1) and Lemma 2.2 that

$$
L_{n, k_{n}} \geq r^{(l-1) \alpha+a-l} C_{n, k_{n}} \geq u_{0} r^{(l-1) \alpha+a-l}(r+1)^{n} .
$$

Therefore the statement of Theorem 1.3 follows immediately. The proof of Theorem 1.3 is complete.

\section{REFERENCES}

[1] P. Bateman, J. Kalb and A. Stenger, A limit involving least common multiples, Amer. Math. Monthly 109 (2002), 393-394.

[2] B. Farhi, Minoration non triviales du plus petit commun multiple de certaines suites finies d'entiers, C.R. Acad. Sci. Paris, Ser. I 341 (2005), 469-474.

[3] B. Farhi, Nontrivial lower bounds for the least common multiple of some finite sequences of integers, J. Number Theory 125 (2007), 393-411.

[4] B. Farhi, An identity involving the least common multiple of binomial coefficients and its application, Amer. Math. Monthly 116 (2009), 836-839.

[5] B. Farhi and D. Kane, New results on the least common multiple of consecutive integers, Proc. Amer. Math. Soc. 137 (2009), 1933-1939.

[6] D. Hanson, On the product of the primes, Canad. Math. Bull. 15 (1972), 33-37.

[7] S. Hong and W. Feng, Lower bounds for the least common multiple of finite arithmetic progressions, C.R. Acad. Sci. Paris, Ser. I 343 (2006), 695-698.

[8] S. Hong and S.D. Kominers, Further improvements of lower bounds for the least common multiples of arithmetic progressions, Proc. Amer. Math. Soc. 138 (2010), 809-813.

[9] S. Hong and G. Qian, The least common multiple of consecutive arithmetic progression terms, Proc. Edinburgh Math. Soc. 54 (2011), 431-441.

[10] S. Hong, G. Qian and Q. Tan, The least common multiple of sequence of product of linear polynomials, Acta. Math. Hungar. 135 (2012), 160-167.

[11] S. Hong and Y. Yang, Improvements of lower bounds for the least common multiple of finite arithmetic progressions, Proc. Amer. Math. Soc. 136 (2008), 4111-4114.

[12] S. Hong and Y. Yang, On the periodicity of an arithmetical function, C.R. Acad. Sci. Paris, Ser. I 346 (2008), 717-721.

[13] M. Nair, On Chebyshev-type inequalities for primes, Amer. Math. Monthly 89 (1982), 126-129.

[14] G. Qian, Q. Tan and S. Hong, The least common multiple of consecutive terms in a quadratic progression, Bull. Aust. Math. Soc. 86 (2012), 389-404.

Mathematical College, Sichuan University, Chengdu 610064, P.R. China

E-mail address: eugen_woo@163.com

School of Mathematics and Computer Science, Panzhinua University, Panzhihua 617000, P.R. CHINA

E-mail address: tqrmei6@126.com

Mathematical College, Sichuan University, Chengdu 610064, P.R. China and Yangtze Center of Mathematics, Sichuan University, Chengdu 610064, P.R. China

E-mail address: sfhong@scu.edu.cn, s-f.hong@tom.com, hongsf02@yahoo.com 